\title{
THE IMPORTANCE OF SURGICAL STAGING IN THE TREATMENT OF MALIGNANT PLEURAL MESOTHELIOMA
}

Valerie W. Rusch, MD

Ennapadam Venkatraman, $\mathrm{PhD}^{\mathrm{b} \S}$
Objectives: Progress in the therapy of malignant pleural mesothelioma is limited by the lack of an adequate staging system and controversy about prognostic factors. This surgical series was analyzed to determine whether a new TNM staging system proposed by the International Mesothelioma Interest Group and certain prognostic factors could stratify patients in future clinical trials. Methods: Thoracotomy was performed if computed tomographic scans showed resectable tumor confined to one hemithorax. Pleurectomy/decortication was done if visceral pleural tumor was minimal, and extrapleural pneumonectomy was done for more locally advanced disease. Complete resection was defined as no gross residual tumor. Adjuvant therapy was given as required by serial clinical trials. Patients had computed tomographic scans every 3 months until death. Prognostic factors were examined by log-rank and Cox regression analyses. Results: From October 1983 to July 1994, a total of 131 thoracotomies were performed, resulting in 101 resections, 72 of which were complete. Extrapleural pneumonectomy was done in 50 patients and pleurectomy/ decortication in 51. The ratio of men to women was 108:23. Median age was 63 years (range 32 to 80 years). Operative mortality was five of 131 patients (3.8\%), three of 50 in the group having extrapleural pneumonectomy $(6 \%)$. Ninety-five of the 131 tumors were epithelial. Fifty-one of 89 patients (57\%) having node dissections had diseased nodes, $45(50 \%)$ N2. By univariate analysis, type of resection, $T$ and $N$ status, stage, histologic type, and adjuvant therapy, but not gender or age, significantly affected survival. Type of resection, stage, and histologic type were significant in a multivariate analysis. Local recurrence occurred mainly after pleurectomy/decortication, and distant metastases developed after extrapleural pneumonectomy. Conclusions: (1) N2 nodal disease is more frequent than previously reported; (2) the prognostic importance of histologic type is confirmed; (3) both $T$ and $N$ status influence outcome, and the International Mesothelioma Interest Group staging system successfully identifies patients whose prognosis is poor; (4) despite more locally advanced disease in most patients with extrapleural pneumonectomy, that approach provided better local control than pleurectomy/decortication but failed to improve survival because of distant metastatic disease. Contrary to past practice, future clinical trials should stratify for histologic type, must control for TNM stage, and must consider the impact of type of surgical resection on the pattern of relapse. (J ThORAC CARDIOVASC SURG 1996;111:815-26)
From the Thoracic Service, Department of Surgery, ${ }^{\text {a }}$ and the Biostatistics Service, Departments of Epidemiology and Biostatistics, ${ }^{b}$ Memorial Sloan-Kettering Cancer Center, New York, N.Y.

Read at the Seventy-fifth Annual Meeting of The American Association for Thoracic Surgery, Boston, Mass., April 23-26, 1995.

Received for publication April 27, 1995; revisions requested
July 17, 1995; revisions received August 7, 1995; accepted for publication Sept. 15, 1995

Address for reprints: Valerie W. Rusch, MD, Thoracic Service, Department of Surgery, Memorial Sloan-Kettering Cancer Center, 1275 York Ave., New York, NY 10021.

${ }^{\S}$ By invitation.

Copyright (C) 1996 by Mosby-Year Book, Inc.

$0022-5223 / 96 \$ 5.00+0 \quad \mathbf{1 2} / 6 / 70603$ 
$T_{\text {him }}^{\text {he }}$ he management of malignant pleural mesothelioma (MPM) remains a subject of controversy because of our poor understanding of the natural history of this disease and its apparent resistance to standard forms of therapy. Prospective clinical trials evaluating novel treatment strategies for MPM are needed but require an accurate, universally accepted staging system that categorizes patients into relatively homogeneous groups. Since 1976, at least five staging systems for MPM have been proposed. ${ }^{1}$ Two of these include specific descriptors of tumor node metastases (TNM), and the other three are based on a simple stage I through IV classification. ${ }^{2-6}$ None of these systems is completely validated or used uniformly for survival analyses. Recently, the International Mesothelioma Interest Group (IMIG) developed a surgically based TNM staging system that reconciles and updates previous staging systems by taking into consideration emerging information about the impact of $\mathrm{T}$ and $\mathrm{N}$ status on survival. ${ }^{7}$ However, even this new staging system is not fully validated. To evaluate whether it could accurately stratify patients in future clinical trials, we retrospectively applied the IMIG staging system to a large prospective surgical registry in which $\mathrm{T}$ and $\mathrm{N}$ status were carefully assessed and patients serially followed up.

\section{Patients and methods}

All patients entered in this prospective registry had biopsy-proved MPM and had an exploratory thoracotomy done by the same surgeon (V.R.). The pathologic diagnosis was always based on both histologic tumor type and immunohistochemistry. When necessary, electron microscopy was added to confirm the diagnosis. Data collected in the registry included patient name, gender, age, date of operation, preoperative platelet count, type of procedure, extent of resection, tumor histologic type, extent of the primary tumor and presence of nodal metastases, operative morbidity and mortality, type of adjuvant therapy, date and site of first relapse, and dates of latest follow-up or death with disease status.

The primary tumor was considered potentially resectable if preoperative computed tomographic scans of the chest and abdomen did not show extrathoracic disease, clear invasion of the mediastinal organs or chest wall, or extension through the diaphragm. The decision to perform an extrapleural pneumonectomy as opposed to a pleurectomy/decortication for resection was based on the extent of visceral pleural tumor at thoracotomy. Extrapleural pneumonectomy, defined as an en-bloc resection of the pleura, lung, ipsilateral diaphragm, and pericardium, ${ }^{8}$ was performed for locally advanced disease, usually in patients with confluent visceral pleural tumor not separable from the lung and a partially or totally fused pleural space. Pleurectomy/decortication, which removes all gross tumor without removing the underlying lung, was performed in patients who had minimal visceral pleural tumor. Partial parietal pleurectomy was sometimes performed for control of a pleural effusion if incompletely resectable tumor was found at exploration, but all pleurectomy/decortications and extrapleural pneumonectomies were performed only if it was thought that all gross tumor could be removed. Complete resection was defined as no gross residual tumor. Resection was defined as incomplete if any visible gross tumor remained at the completion of thoracotomy, even if only a few scattered tumor foci less than $5 \mathrm{~mm}$ in size were present.

The recently developed IMIG staging system (Table I) was applied retrospectively to each patient to determine his or her TN status and corresponding tumor stage. Staging was based on precise information about tumor extent in the operative summary dictated by the attending surgeon and on nodal involvement as recorded in the pathology report.

Many of the patients entered in this registry were also enrolled in three sequential prospective multisurgeon or multiinstitutional clinical trials. ${ }^{9-11}$ Postoperative adjuvant therapy, if any, was determined by those trial protocols. Adjuvant chemotherapy was given only in the protocol setting. Patients not entered on a clinical trial usually received postoperative hemithoracic radiation. The total radiation dose and method of administration depended on whether a pneumonectomy was performed.

Patients in whom thoracotomy disclosed unresectable tumor were followed up clinically until death. Patients who had a pleurectomy/decortication or extrapleural pneumonectomy were followed up by physical examination and computed tomographic scans of the chest and abdomen every 3 months until the time of first recurrence. Additional imaging studies or biopsies were performed as necessary to document recurrent disease. Thereafter, these patients were also followed up clinically until the time of death. Thus accurate information is available about the first site of relapse in these patients, but not about all subsequent sites of relapse.

Survival probabilities were calculated by the product limit method of Kaplan and Meier. ${ }^{12}$ The prognostic significance of factors was tested in a univariate model by the log-rank statistic for categoric covariates ${ }^{13}$ and by proportional hazards regression for continuous covariates. ${ }^{14}$ Proportional hazards regression was used to test the prognostic significance of factors in a multivariate model. The $p$ value was considered significant when it was less than 0.05 .

\section{Results}

From October 1983 to July 1994, 131 consecutive patients with MPM underwent exploratory thoracotomy, including 108 men and 23 women with a median age of 63 years (range 32 to 80 years). A total of $101(77 \%)$ resections were performed, including 50 extrapleural pneumonectomies and 51 pleurectomy/decortications. The resection was considered complete in 72 patients ( $71 \%$ of resections, $55 \%$ of all thoracotomies). The 30 -day operative 
Table I. New international staging system for diffuse malignant pleural mesothelioma, developed by the International Mesothelioma Interest Group

\section{$\mathbf{T}=$ Tumor}

T1

T1a Tumor limited to the ipsilateral parietal including mediastinal and diaphragmatic pleura No involvement of the visceral pleura

T1b Tumor involving the ipsilateral parietal including mediastinal and diaphragmatic pleura Scattered foci of tumor also involving the visceral pleura

T2 Tumor involving each of the ipsilateral pleural surfaces (parietal, mediastinal, diaphragmatic, and visceral pleura) with at least one of the following features:

- Involvement of diaphragmatic muscle

- Confluent visceral pleural tumor (including the fissures), or extension of tumor from visceral pleura into the underlying pulmonary parenchyma

T3 Describes locally advanced but potentially resectable tumor

Tumor involving all of the ipsilateral pleural surfaces (parietal, mediastinal, diaphragmatic, and visceral pleura) with at least one of the following features:

- Involvement of the endothoracic fascia

- Extension into the mediastinal fat

- Solitary, completely resectable focus of tumor extending into the soft tissues of the chest wall

- Nontransmural involvement of the pericardium

T4 Describes locally advanced technically unresectable tumor

Tumor involving all of the ipsilateral pleural surfaces (parietal, mediastinal, diaphragmatic, and visceral) with at least one of the following features:

- Diffuse extension or multifocal masses of tumor in the chest wall, with or without associated rib destruction

- Direct transdiaphragmatic extension of tumor to the peritoneum

- Direct extension of tumor to the contralateral pleura

- Direct extension of tumor to one or more mediastinal organs

- Direct extension of tumor into the spine

- Tumor extending through to the internal surface of the pericardium with or without a pericardial effusion; or tumor involving the myocardium

\section{$\mathbf{N}=$ Lymph nodes}

NX Regional lymph nodes cannot be assessed

No No regional lymph node metastases

N1 Metastases in the ipsilateral bronchopulmonary or hilar lymph nodes

N2 Metastases in the subcarinal or the ipsilateral mediastinal lymph nodes including the ipsilateral internal thoracic nodes

N3 Metastases in the contralateral mediastinal, contralateral internal thoracic, ipsilateral, or contralateral supraclavicular lymph nodes

\section{$\mathbf{M}=$ Metastases}

MX Presence of distant metastases cannot be assessed

M0 No distant metastasis

M1 Distant metastasis present

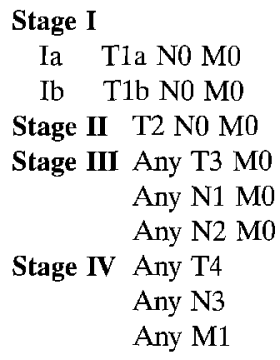

mortality rate was $3.8 \%(5 / 131$ patients) and was $6 \%$ (3/50 patients) among the patients having extrapleural pneumonectomy. The causes of death in the two patients who had a pleurectomy/decortication were a cerebrovascular accident and multisystem failure related to massive bleeding in the upper gastrointestinal tract from previously unsuspected peptic ulcer disease. The causes of death in the three patients who had an extrapleural pneumonectomy were massive pulmonary embolus, respiratory fail- 
Table II. Postoperative complications in all 131 patients with MPM who underwent exploratory thoracotomy

\begin{tabular}{|c|c|c|c|c|}
\hline \multirow[b]{2}{*}{$\begin{array}{c}\text { Type of } \\
\text { complication }\end{array}$} & \multicolumn{3}{|c|}{$\begin{array}{l}\text { No. of patients experiencing complication } \\
\text { according to type of procedure }\end{array}$} & \multirow[b]{2}{*}{$\begin{array}{l}\text { Total no. of } \\
\text { complications }\end{array}$} \\
\hline & $\begin{array}{l}\text { Extrapleural pneumonectomy } \\
\qquad(n=18)\end{array}$ & $\begin{array}{l}\text { Pleurectomy/decortication } \\
\qquad(n=13)\end{array}$ & $\begin{array}{l}\text { Other } \\
(n=2)\end{array}$ & \\
\hline Atrial arrhythmia & 11 & 2 & 1 & 14 \\
\hline Empyema & 2 & 4 & 0 & 6 \\
\hline Postoperative death & 3 & 1 & 1 & 5 \\
\hline Prolonged chest tube air leak & 0 & 5 & 0 & 5 \\
\hline Pneumonia & 3 & 0 & 0 & 3 \\
\hline Pulmonary embolus & 1 & 1 & 0 & 2 \\
\hline Respiratory failure & 2 & 0 & 0 & 2 \\
\hline Multisystem failure & 1 & 1 & 0 & 2 \\
\hline Renal failure (self-limited) & 1 & 1 & 0 & 2 \\
\hline Ventricular arrhythmia & 0 & 1 & 0 & 1 \\
\hline
\end{tabular}

The number of complications is shown according to the type of surgical procedure performed. Because some patients had more than one complication, for example, bronchial stump leak and empyema, the total number of complications listed exceeds the number of patients. Eighteen of the extrapleural pneumonectomy group $(36 \%)$ and 13 of the pleurectomy/decortication group $(25.5 \%)$ had complications. Two patients undergoing other procedures had complications $(7 \%)$. Ninety-eight patients had no complications.

ure related to underlying interstitial lung disease, and respiratory failure from fulminant pneumonia related to underlying diabetes mellitus.

The postoperative complications are shown in Table II and occurred in 33 of the $131(25 \%)$ patients. The most common complication was atrial arrhythmia, which occurred in 11 of the patients having extrapleural pneumonectomy (22\%) and two of those having pleurectomy/decortication (4\%).

According to the IMIG staging system, 18 patients had T1 tumors, whereas 36 had T2, 45 had T3, and 32 had T4 tumors. Mediastinal lymph node dissection was performed in 89 of the $101(88 \%)$ patients undergoing resection. Fifty-one of these patients (57\%) had nodal metastases and $45(50 \%)$ had these metastases in the ipsilateral mediastinal lymph nodes (N2 disease). One patient had N3 disease and five patients had only $\mathrm{N} 1$ disease. In 95 of the $131(76 \%)$ patients, the histologic tumor type was epithelial, whereas it was fibrosarcomatous in eight $(6 \%)$ patients, of mixed cellularity in $26(20 \%)$ patients, and desmoplastic in one patient. The histologic subtype was not specified in one patient. The preoperative platelet count was not uniformly recorded during the first 5 years of this registry. It was known in 121 patients, of whom 41 were considered to have thrombocytosis because of a platelet count exceeding $400,000 / \mathrm{mm}^{3}$.

All 131 patients were included in the analyses of survival rates. The median follow-up for all 131 patients was 9.4 months, 8.8 months for those having extrapleural pneumonectomy and 16.8 months for those having pleurectomy/decortication. The rela- tively short length of follow-up reflects the poor overall survivals in these patients, because they are all followed up until death. The median survival for patients categorized according to the type of surgical resection, the tumor $\mathrm{T}$ and $\mathrm{N}$ status, stage, and histologic type is shown in Table III. The overall survivals according to these same variables are shown in Figs. 1 to 5. In univariate analyses the patients undergoing pleurectomy/decortication had a significantly better survival than did those undergoing extrapleural pneumonectomy $(p=0.0054)$, and both of these groups survived significantly longer than did patients who had a palliative pleurectomy or only an exploratory thoracotomy (Fig. 1). The differences in survival according to $T$ status (Fig. 2) were assessed by log-rank test across all $\mathrm{T}$ categories and were found to be significant ( $p=$ 0.0058 ). Because of the small numbers of patients in each group, individual comparisons are inappropriate; hence T1/T2 was compared with T3/T4. Patients with T1/T2 status had a significantly better survival $(p=0.0079)$. Likewise, the small number of patients with either N1 or N3 disease necessitated that patients having $\mathrm{N} 0$ disease be compared with those having N1-3 disease. Patients with N0 disease had a significantly better survival than did those with nodal metastases $(p=0.0074)$, in this case predominantly ipsilateral mediastinal nodal metastases (Fig. 3). Because the overall and median survivals for $T 2$ and T3 tumors appeared relatively similar, these categories were further examined by $\mathrm{N}$ status (N0 versus N1-3). No significant differences were detected in overall survivals for $\mathrm{T} 2 \mathrm{~N} 0$ versus $\mathrm{T} 3 \mathrm{~N} 0$ 
$(p=0.77)$, for $\mathrm{T} 2 \mathrm{~N} 1-3$ versus $\mathrm{T} 3 \mathrm{~N} 1-3(p=0.33)$, for T2 N0 versus T2 N1-3 $(p=0.28)$, or for T3 N0 versus T3 N1-3 $(p=0.07)$. However, as shown in Fig. 4, a trend toward worsening survival is related to both $\mathrm{T}$ and $\mathrm{N}$ status. The results of this analysis must be interpreted with caution because the number of patients in these TN subsets is too small to yield statistically definitive results.

The differences in survival by stage (Fig. 5) were assessed by log-rank test across all four stages and were found to be significant $(p=0.001)$. Fig. 5 shows a trend of decreasing survival by stage, although individual comparisons between stages (for $p$ value) are not justified by virtue of the small number of patients in each stage category. When stage I and II were grouped together they were associated with a significantly better survival than stages III and IV $(p=0.0001)$. Epithelial tumors were associated with a significantly better survival $(p=0.0002)$ than were tumors of other histologic types (Fig. 6). Thrombocytosis did not have an adverse effect on survival (Fig. 7).

Patients who received any form of postoperative adjuvant therapy $(n=93)$ were compared in a univariate analysis with those who did not, and overall survival was significantly better in the former group. No significant difference in overall survival could be detected according to the type of adjuvant therapy, but the variation in treatment and the resultant small number of patients in each treatment category preclude a meaningful analysis. Univariate analyses also showed no significant difference in overall survival between complete and incomplete resection, between age less or greater than 50 or than 60 years, or between men and women.

When a multivariate analysis was performed, only tumor stage $(p=0.0063)$, histologic type $(p=$ 0.0064), and type of surgical procedure still had a significant influence on overall survival. Although still significant, the difference in survival between pleurectomy/decortication and extrapleural pneumonectomy was diminished in a multivariate analysis $(p=0.022)$.

The sites of relapse in the 101 patients who had either an extrapleural pneumonectomy or a pleurectomy/decortication are shown in Table IV. Forty-six patients had a relapse in a single disease site, and 25 had recurrent disease in multiple sites. Recurrences in the ipsilateral chest wall and mediastinum and in the region of previously resected pleura were seen in a few patients treated by extrapleural pneumonectomy but were far more common among those
Table III. Median survival for all 131 patients according to TN status, stage, and histologic type

\begin{tabular}{lc}
\hline & Median survival (mo) \\
\hline Operation & \\
Extrapleural pneumonectomy & 9.9 \\
Pleurectomy/decortication & 18.3 \\
T status & \\
T1 & 27 \\
T2 & 12 \\
T3 & 13 \\
T4 & 6.5 \\
N status & \\
N0 & 18.3 \\
N1-3 & 9.4 \\
Stage & \\
I & 35 \\
II & 16 \\
III & 11.5 \\
IV & 5.9 \\
Histologic type & \\
Epithelial & 15.1 \\
Nonepithelial & 6 \\
\hline
\end{tabular}

The median survival for the 101 patients who had either a pleurectomy/ decortication or extrapleural pneumonectomy is shown according to which operation was performed.

having pleurectomy/decortication. The ipsilateral pleura was the single most common site of recurrent tumor after pleurectomy/decortication. In contrast, relapse in distant areas was more frequent after extrapleural pneumonectomy and occurred particularly in the contralateral pleura and lung, intraabdominal organs, and peritoneum.

\section{Discussion}

For many years MPM was considered a rare and idiopathic cancer. Study of this disease did not begin in earnest until 1960, when Wagner, Slegg, and Marchand ${ }^{15}$ established the link between asbestos exposure and the subsequent development of MPM. The treatment of MPM has gained importance because it is becoming more prevalent worldwide and is still invariably fatal. ${ }^{16}$ During the past 30 years, our understanding of the epidemiology and pathology of MPM has been steadily improving. ${ }^{17-20}$ However, our understanding of its natural history is still incomplete. The seemingly capricious behavior of MPM and its relative resistance to standard forms of cancer therapy often engender a sense of nihilism and an ad hoc approach to treatment. ${ }^{21-25}$

The critical importance of an accurate staging system and of identifying significant prognostic factors is well accepted in the study and treatment of all other solid tumors. In MPM, some prognostic fac- 


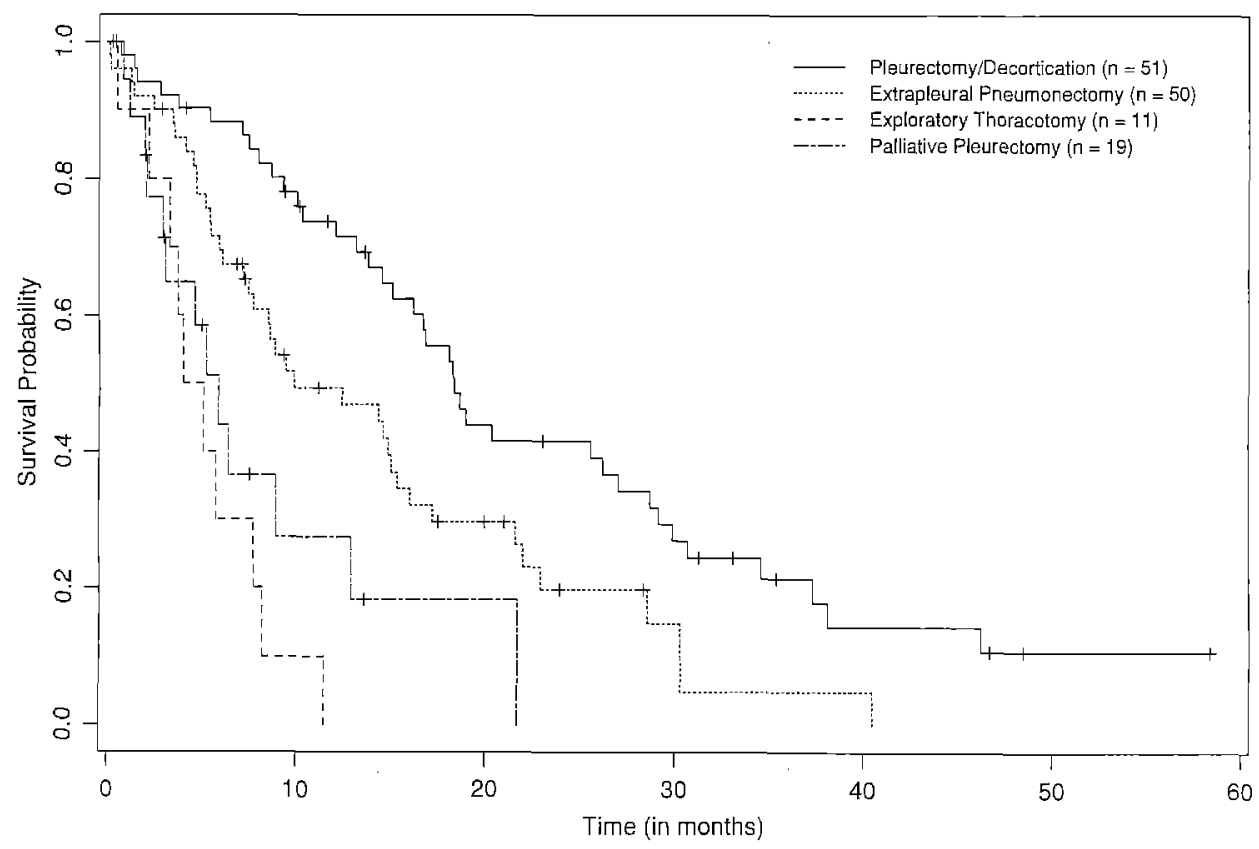

Fig. 1. Univariate analysis of overall survival according to type of surgical procedure performed. When pleurectomy/decortication was compared with extrapleural pneumonectomy, $p$ was 0.0054 .



Fig. 2. Univariate analysis of overall survival by $\mathrm{T}$ status. When $\mathrm{T} 1 / \mathrm{T} 2$ was compared with $\mathrm{T} 3 / \mathrm{T} 4, p$ was 0.0079 . When all four $T$ groups were compared simultaneously, $p$ was 0.0058 .

tors have been identified, but no universally accepted staging system exists. During the past 20 years, at least five systems have been proposed. ${ }^{2-6}$ None is fully validated or consistently used for survival analyses. The recently developed IMIG staging system reconciles and updates earlier staging systems and provides precise TNM descriptors that can be used for radiographic, surgical, and patho- 


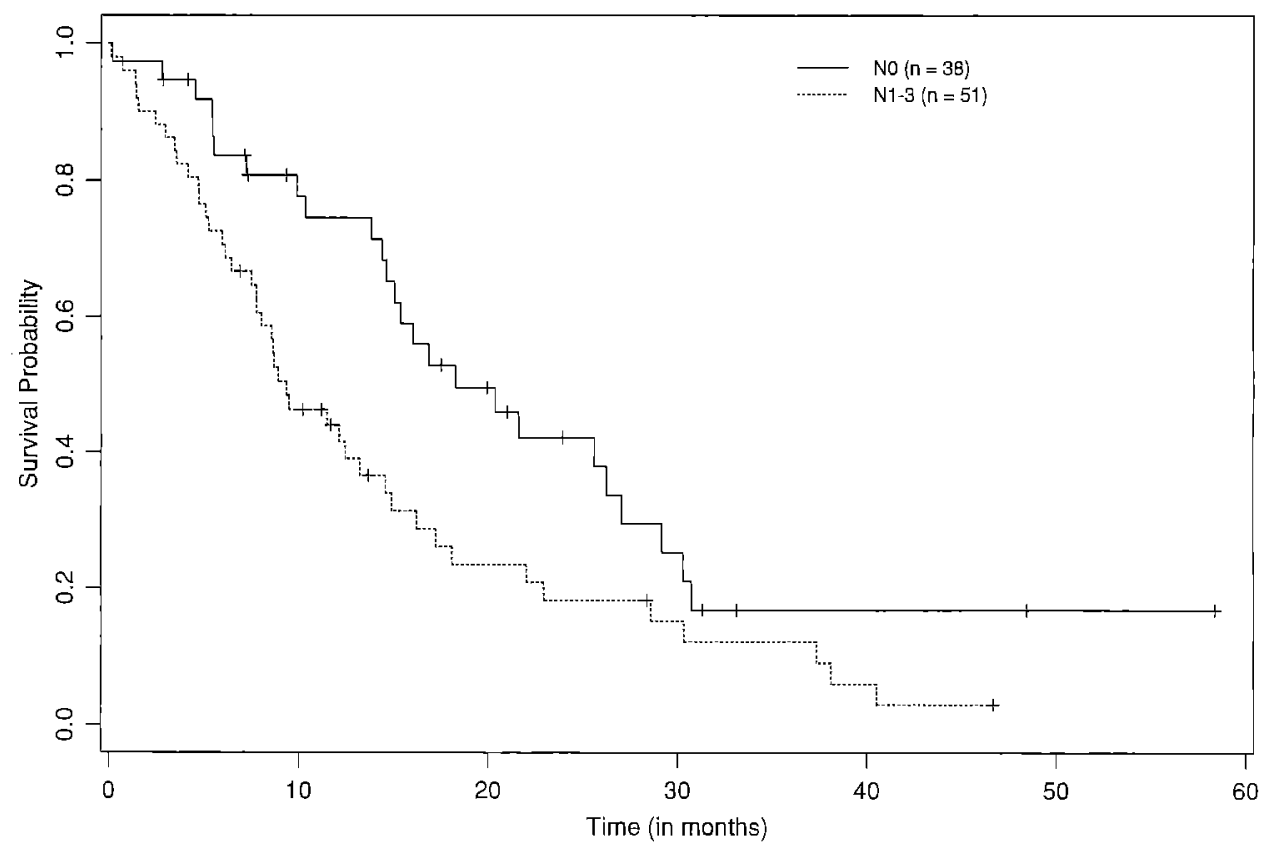

Fig. 3. Univariate analysis of overall survival by $\mathrm{N}$ status. When $\mathrm{N} 0$ was compared with $\mathrm{N} 1-3, p$ was 0.0074 .

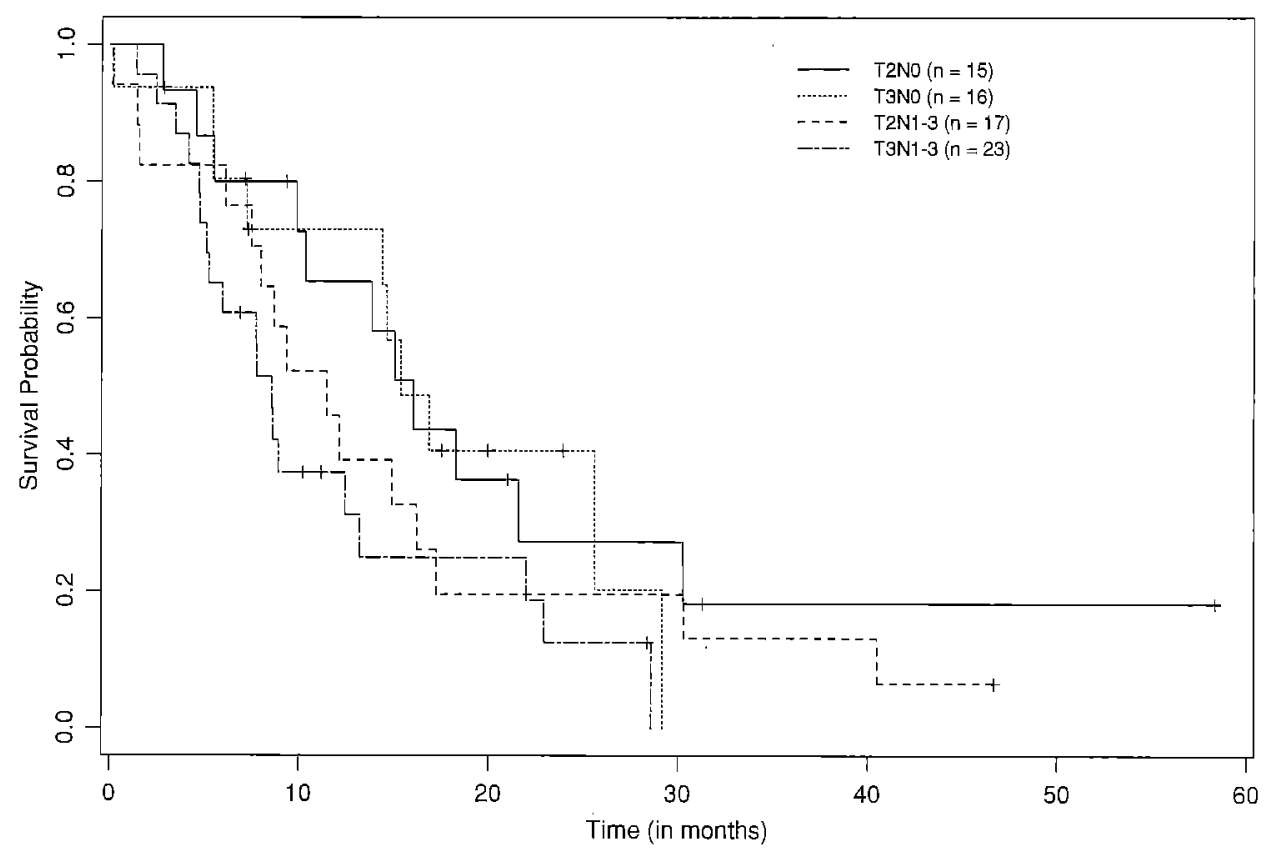

Fig. 4. Univariate analysis of overall survival of $\mathrm{T} 2$ and $\mathrm{T} 3$ analyzed by $\mathrm{N}$ status. Because almost all patients with known nodal metastases had N2 nodes involved, these are grouped together as N1-3.

logic staging of MPM. Although based on emerging information about the impact of $\mathrm{T}$ and $\mathrm{N}$ status on survival, the IMIG system is also not fully validated by large surgical series of patients with carefully staged disease. In particular, the adverse influence of nodal metastases on survival is reported in one surgical series ${ }^{3}$ but not in others. ${ }^{26}$ Our experience indicates that the IMIG staging system does stratify 




Fig. 5. Univariate analysis of overall survival by stage. When stage I/II was compared with stage III/IV, $p$ was 0.0001 . When all four stage groups were compared simultaneously, $p$ was also 0.0001 .

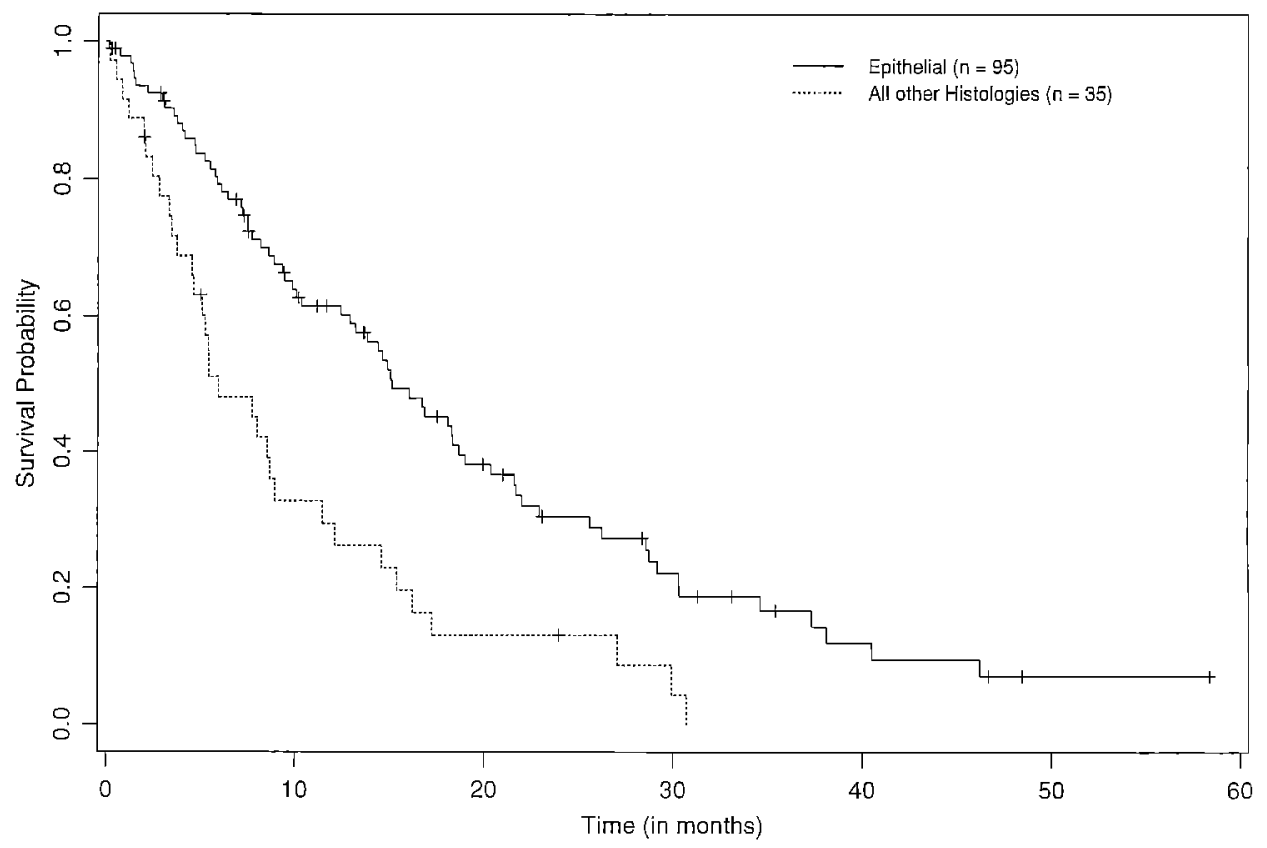

Fig. 6. Univariate analysis of overall survival by tumor histology. Epithelial histology was associated with a significantly better survival than all other histologic types $(p=0.0002)$.

patients appropriately according to prognosis, and it confirms the adverse influence of both advanced $T$ status and nodal metastases on overall survival. Moreover, the frequency of $\mathbf{N} 2$ disease in this series is higher than reported previously ${ }^{3}$ and suggests that complete mediastinal lymph node dissection or sampling is important in accurately determining disease stage. 


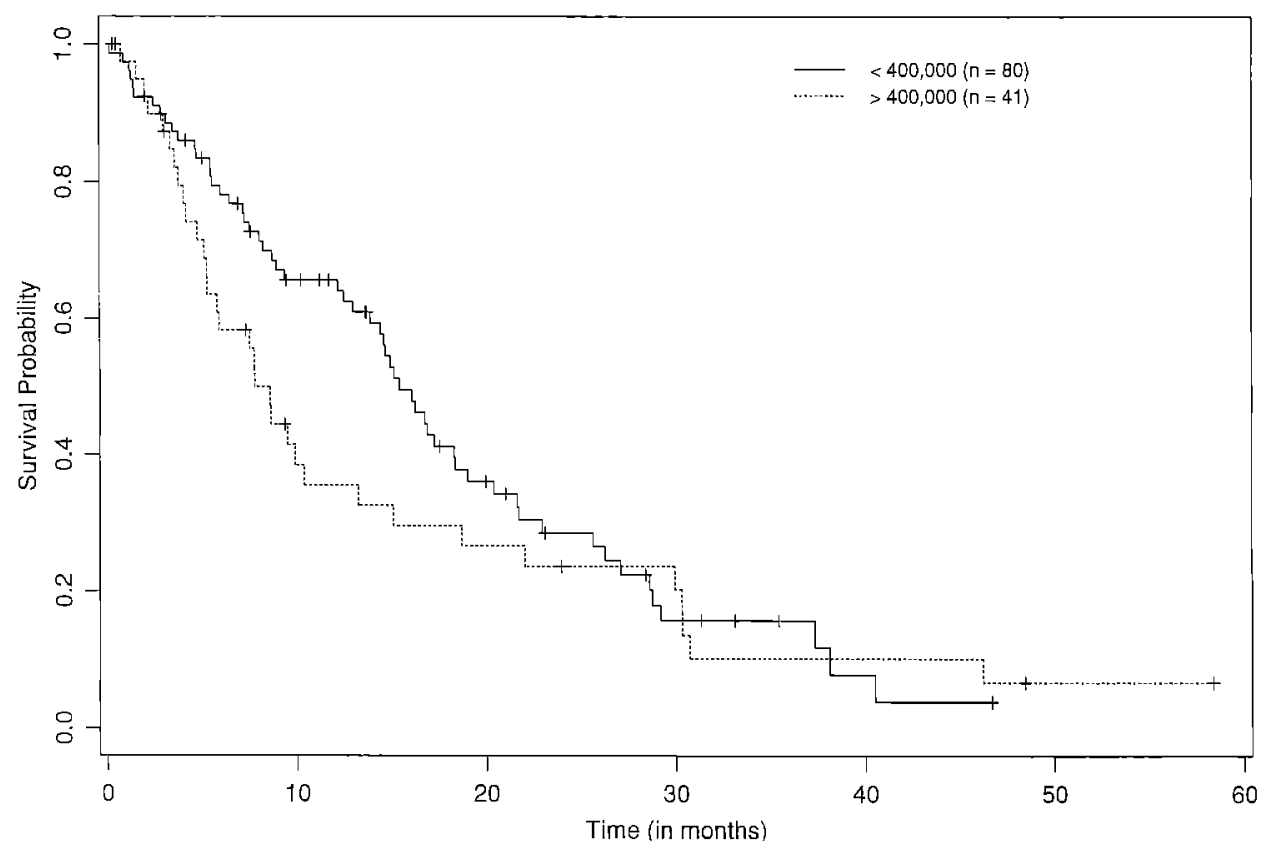

Fig. 7. Univariate analysis of overall survival by preoperative platelet count. Thrombocytosis does not have a significant effect on survival $(p>0.05)$.

The systematic use of a staging system such as the IMIG system could help resolve the controversies surrounding the treatment of MPM. For instance, some authors argue that patients with MPM should receive only supportive care ${ }^{21,23,24}$ because the natural history of MPM is variable and because no therapeutic modality appears to improve life expectancy. Yet recent data, particularly from the thoracoscopic experience of Boutin and associates, ${ }^{27,28}$ suggest that the variation in survivals reported with supportive care alone is largely explained by the stage of disease at diagnosis and the histologic tumor type. Grouping together all patients with MPM regardless of these factors is analogous to grouping together patients with $\mathrm{T} 1 \mathrm{~N} 0$ and stage IV non-small-cell lung cancer. No therapeutic modality can be properly assessed unless applied to groups of patients with a relatively homogeneous prognosis.

The routine use of a TNM staging system could also resolve controversy over the choice of operation for patients who have potentially resectable MPM. For patients with more locally advanced tumor and extensive visceral pleural disease, only extrapleural pneumonectomy allows complete resection of all gross disease. However, for patients with minimal visceral pleural tumor, pleurectomy/ decortication was advocated as a safer operation
Table IV. First sites of recurrent disease

\begin{tabular}{lrrrrr}
\hline & $\begin{array}{c}\text { Single site } \\
(N=46)\end{array}$ & & \multicolumn{2}{c}{$\begin{array}{c}\text { Multiple sites } \\
(N=25)\end{array}$} \\
\cline { 2 - 3 } \cline { 5 - 6 } \multicolumn{1}{c}{ Site of relapse } & $E P P$ & $P / D$ & & $E P P$ & $P / D$ \\
\hline Ipsilateral "pleura" & 2 & 18 & & 3 & 7 \\
Contralateral pleura & 6 & 2 & & 0 \\
Ipsilateral lung & - & 2 & & - & 1 \\
Contralateral lung & 3 & 0 & & 1 \\
Pericardium & 1 & 0 & & 0 \\
Myocardium & 0 & 0 & & 0 \\
Mediastinum & 0 & 0 & & 6 & 2 \\
Chest wall & 2 & 3 & & 6 & 2 \\
Intra-abdominal, visceral & 3 & 0 & & 3 \\
Peritoneum & 2 & 0 & & 3 & 5 \\
Central nervous system & 0 & 0 & 0 & 0 \\
Retroperitoneum & 1 & 0 & & 1 & 0 \\
Other & 0 & 1 & & 0 \\
\hline
\end{tabular}

First sites of relapse in the 71 patients who underwent either extrapleural pneumonectomy (EPP) or pleurectomy/decortication (P/D) and then had recurrent disease. Forty-six patients had a relapse in a single disease site and 25 patients had relapses in multiple sites. The sites of relapse are displayed according to the type of surgical resection performed.

than extrapleural pneumonectomy, which previously had a reported mortality rate of $15 \%$ to $30 \% ., 9$ Recent experience showing that the mortality rate of extrapleural pneumonectomy can be reduced to less than $10 \%{ }^{26,29}$ has further fueled this controversy. Another aspect of this controversy is that the choice of operation has important implications for the use 
of adjuvant postoperative radiation. Hemithoracic radiation can be administered to a higher total dose after extrapleural pneumonectomy than after pleurectomy/decortication. ${ }^{30,31}$ If a treatment regimen of surgical resection and postoperative radiation is planned, pleurectomy/decortication in patients with very early tumor offers a lower early mortality but potentially less effective adjuvant therapy, whereas extrapleural pneumonectomy offers a higher early mortality but potentially more effective radiotherapy.

Our experience confirms that the operative mortality of extrapleural pneumonectomy, although still higher than for a pleurectomy/decortication, is similar to that of a standard pneumonectomy in the hands of surgeons or single institutions that perform this operation frequently. However, when considered solely in a univariate analysis, pleurectomy/ decortication in this series was associated with a better overall survival than extrapleural pneumonectomy. The application of a multivariate analysis to survival suggests that the type of surgical resection is much less important when considered in the context of stage and histology. Previous attempts to determine the relative benefit of extrapleural pneumonectomy versus pleurectomy/decortication have not truly taken TN status into account. Our experience emphasizes the importance of controlling for stage and histology when assessing surgical results.

Our data also corroborate a previous report from the Lung Cancer Study Group ${ }^{9}$ suggesting that distant metastases may be the initial site of relapse more frequently after extrapleural pneumonectomy than after pleurectomy/decortication. However, this could also reflect the disease stage and prevalence of nodal metastases. These findings need to be examined further in larger numbers of patients in whom the disease has been carefully staged.

No TNM staging system fully recognizes the pathologic and biologic variables that influence survival. Many factors are reported to be prognostic in MPM, including histologic type, age, gender, performance status, length of time to diagnosis, type of symptoms, completeness of surgical resection, weight loss, history of asbestos exposure, and platelet count. ${ }^{28,32-36^{*}}$ Some purported prognostic factors including the type of symptoms, weight loss, and performance status reflect tumor stage and were not examined in this analysis. No effect of age or gender on survival was found in our experience, but the influence of gender is difficult to assess because of

* Chahian AP: Personal communication. the small number of women in this series. The lack of effect of complete resection on overall survival could be related to the stringent definition used in this series. Incomplete resection described patients who had minimal gross residual tumor, usually scattered islands of tumors less than $5 \mathrm{~mm}$ in size. Patients who would have had more extensive residual tumor were simply not offered resection with curative intent. Importantly, our experience confirms the beneficial impact of epithelial histology, which has been the most consistent prognostic factor across all reported series.

In summary, this experience with patients in whom the extent of disease was carefully staged at thoracotomy suggests that the new IMIG staging system for MPM accurately classifies patients according to prognosis. Current information about the impact of N1 versus N2 nodal metastases is inadequate to distinguish between involvement of these two nodal groups. In this series, N1 nodes in the resected specimen were not always specifically examined by the pathologist; therefore the true prevalence of N1 disease is unknown. It is possible that N2 nodes are involved earlier or more frequently in MPM because the tumor arises in the parietal pleura and involves the entire pleural space. The differential influence of $\mathrm{N} 1$ and $\mathrm{N} 2$ nodes should be examined in future surgical series. The importance of histologic type is confirmed and suggests that patients should be stratified according to whether they have epithelial tumors or one of the other histologic subtypes of MPM. Additional careful clinicopathologic correlation in large prospective surgical series is needed to test the validity of the IMIG staging system and to determine whether the $\mathrm{T}$ and $\mathrm{N}$ descriptors should be revised or combined into broader stage classifications. For example, it is possible that the T2 and T3 categories will prove to have overall survivals that are sufficiently similar to warrant collapsing these descriptors into a single group. Likewise, much more information is needed about the prevalence of N1 and N3 nodal metastases and the influence of these nodal groups on outcome. However, at present the analysis of this series suggests that future clinical trials should include careful TNM staging with the IMIG system and stratification by histologic type to assess whether new treatment strategies alter overall survival.

We thank Melody Owens for her assistance in data management and the preparation of this manuscript. 


\section{REFERENCES}

1. Dimitrov NV, McMahon S. Presentation, diagnostic methods, staging, and natural history of malignant mesothelioma. In: Antman K, Aisner J, eds. Asbestos-related malignancy. Orlando: Grune \& Stratton, 1987:225-38.

2. Chahinian AP. Therapeutic modalities in malignant pleural mesothelioma. In: Chretien J, Hirsch A, eds. Diseases of the pleura. New York: Masson, 1983:224-36.

3. Sugarbaker DJ, Strauss GM, Lynch TJ, et al. Node status has prognostic significance in the multimodality therapy of diffuse, malignant mesothelioma. J Clin Oncol 1993;11:1172-8.

4. Butchart EG, Ashcroft T, Barnsley WC, Holden MP. Pleuropneumonectomy in the management of diffuse malignant mesothelioma of the pleura: experience with 29 patients. Thorax 1976;31:15-24.

5. Mattson K. Natural history and clinical staging of malignant mesothelioma [Abstract]. Eur J Respir Dis 1982;63(Suppl 124):87.

6. Rusch VW, Ginsberg RJ. New concepts in the staging of mesotheliomas. In: Deslauriers J, Lacquet LK, eds. Thoracic surgery: surgical management of pleural diseases. St. Louis: Mosby, 1990:336-43.

7. Rusch VW, The International Mesothelioma Interest Group. a proposed new international TNM staging system for malignant pleural mesothelioma. Chest [In press].

8. Rusch VW, Dillard D. Pleural mesothelioma. In: Pearson FG, Deslauriers J, Ginsberg RJ, Hiebert CA, McKneally MF, Urschel HC, eds. Thoracic surgery. New York: Churchill Livingstone, 1995:1083-105.

9. Rusch VW, Piantadosi S, Holmes EC. The role of extrapleural pneumonectomy in malignant pleural mesothelioma. $\mathbf{J}$ Thorac Cardiovasc Surg 1991;102:1-9.

10. Rusch VW, Saltz L, Venkatraman E, et al. A phase II trial of pleurectomy/decortication followed by intrapleural and systemic chemotherapy for malignant pleural mesothelioma. J Clin Oncol 1994;12:1156-63.

11. Rusch V, Livingston R. Radical decortication, intraoperative intrapleural cisplatin (CDDP) and post-operative systemic chemotherapy for malignant pleural mesothelioma (MM) [Abstract]. Proc Am Soc Clin Oncol 1989;8:219.

12. Kaplan EL, Meier P. Nonparametric estimation from incomplete observations. J Am Stat Assoc 1958;53:457-81.

13. Mantel N, Haenszel W. Statistical aspects of the analysis of data from retrospective studies of disease. J Natl Cancer Inst 1958;22:718-48.

14. Cox DR. Regression models and life tables [with discussion]. J R Stat Soc B 1972;34:187-220.

15. Wagner JC, Slegg CA, Marchand P. Diffuse pleural mesotheliomas and asbestos exposure in Northwestern Cape Province. Br J Ind Med 1960;17:260-71.

16. Spirtas R, Connelly RR, Tucker MA. Survival patterns for malignant mesothelioma: the SEER experience. Int $\mathrm{J}$ Cancer 1988;41:525-30.

17. Wagner JC. Mesothelioma and mineral fibers. Cancer 1986; 57:1905-11

18. Adams VI, Unni KK, Muhm JR, Jett JR, Ilstrup DM, Bernatz PE. Diffuse malignant mesothelioma of pleura: diagnosis and survival in 92 cases. Cancer 1986;58:1540-51.

19. Nauta RJ, Osteen RT, Antman KH, Koster JK. Clinical staging and the tendency of malignant pleural mesotheliomas to remain localized. Ann Thorac Surg 1982;34:66-70.

20. Wirth PR, Legier J, Wright GL Jr. Immunohistochemical evaluation of seven monoclonal antibodies for differentiation of pleural mesothelioma from lung adenocarcinoma. Cancer 1991;67:655-62.

21. Law MR, Gregor A, Hodson ME, Bloom HJG, Turner-Warwick M. Malignant mesothelioma of the pleura: a study of 52 treated and 64 untreated patients. Thorax 1984;39:255-9.

22. Lewis RJ, Sisler GE, Mackenzie JW. Diffuse, mixed malignant pleural mesothelioma. Ann Thorac Surg 1981;31:53-60.

23. Alberts AS, Falkson G, Goedhals L, Vorobiof DA, Van Der Merwe CA. Malignant pleural mesothelioma: a disease unaffected by current therapeutic maneuvers. J Clin Oncol 1988;6:527-35.

24. Achatzy R, Beba W, Ritschler R, et al. The diagnosis, therapy and prognosis of diffuse malignant mesothelioma. Eur $\mathbf{J}$ Cardiothorac Surg 1989;3:445-8.

25. Hulks G, Thomas JStJ, Waclawski E. Malignant pleural mesothelioma in western Glasgow 1980-6. Thorax 1989;44: 496-500.

26. Allen KB, Faber LP, Warren WH. Malignant pleural mesothelioma: extrapleural pneumonectomy and pleurectomy. Chest Surg Clin North Am 1994;4:113-26.

27. Boutin C, Rey F. Thoracoscopy in pleural malignant mesothelioma: a prospective study of 188 consecutive patients. Part 1: Diagnosis. Cancer 1993;72:389-93.

28. Boutin C, Rey F, Gouvernet J, Viallat J-R, Astoul P, Ledoray $\mathrm{V}$. Thoracoscopy in pleural malignant mesothelioma: a prospective study of 188 consecutive patients. Part 2: Prognosis and staging. Cancer 1993;72:394-404.

29. Sugarbaker DJ, Heher EC, Lee TH, et al. extrapleural pneumonectomy, chemotherapy, and radiotherapy in the treatment of diffuse malignant pleural mesothelioma. J THORaC Cardiovasc Surg 1991;102:10-5.

30. Maasilta P. Deterioration in lung function following hemithorax irradiation for pleural mesothelioma. Int J Radiation Oncol Biol Physiol 1991;20:433-8.

31. Mychalczak BR, Nori D, Armstrong JG, Martini N, Harrison LB. Results of treatment of malignant pleural mesothelioma with surgery, brachytherapy, and external beam irradiation [Abstract]. Proceedings of the American Endocurietherapy Society $1989 ; 12$.

32. Tammilehto L. Malignant mesothelioma: prognostic factors in a prospective study of 98 patients. Lung Cancer 1992;8: 175-84.

33. Pogrebniak H, Temeck B, Steinberg S, Kranda K, Pass H. Cisplatin, interferon, and tamoxifen for primary and adjuvant use in malignant pleural mesothelioma. Ann Surg Oncol [In press].

34. Ruffie R, Feld R, Minkin S, et al. Diffuse malignant mesothelioma of the pleura in Ontario and Quebec: a retrospective study of 332 patients. J Clin Oncol 1989;7:1157-68.

35. Antman KH, Shemin R, Ryan L, et al. Malignant mesothelioma: prognostic variables in a registry of 180 patients, the Dana-Farber Cancer Institute and Brigham and Women's Hospital experience over two decades, 1965-1985. J Clin Oncol 1988;6:147-53.

36. Chahinian AP, Pajak TF, Holland JF, Norton L. Diffuse malignant mesothelioma: prospective evaluation of $69 \mathrm{pa}-$ tients. Ann Intern Med 1982;96(6, Pt 1):746-55.

\section{Discussion}

Dr. Douglas J. Mathisen (Boston, Mass.). Can you explain the difference between palliative pleurectomy and pleurec- 
tomy/decortication? There was a survival difference, and I think it would be important to explain this difference.

Dr. Rusch. Patients who had a pleurectomy/decortication with therapeutic intent had all or almost all gross tumor removed. Patients who had a "palliative" pleurectomy had an exploratory operation in which the intent was to resect, but the tumor could not be completely removed and only a parietal pleurectomy was done to control the effusion. Thus "palliative" pleurectomy was a much less extensive resection.

Dr. Malcolm M. DeCamp (Boston, Mass.). Your paper underscores one of the universal problems in mesothelioma-trying to determine who should receive aggressive surgical therapy and who should receive palliative therapy with less invasive techniques. In this retrospective series, how hard was it to get your pathologists to evaluate pleurectomy specimens and assign them specific $\mathrm{T}$ stages? You have carefully defined $\mathrm{T}$ in terms of $1 \mathrm{~A}, 1 \mathrm{~B}, 2$, and so on. Was that difficult? We have a difficult problem getting our pathologists to accurately evaluate depth of invasion at so many different margins.

Dr. Rusch. I think it is difficult, perhaps impossible, for pathologists to examine margins in this disease, although I am aware that at the Brigham there has been an effort to do so. This staging system is primarily designed to stage the disease surgically either at thoracoscopy or thoracotomy. The $\mathrm{T}$ status is established by the surgical findings, not necessarily the pathologic findings. The $\mathrm{N}$ status, of course, is established pathologically.

Dr. Robert J. Ginsberg (New York, N.Y.). It has been about 30 years since the first attempt at staging mesothelioma was made. As Dr. Rusch rightly pointed out, there are six or seven staging systems, and every article espouses a different staging system. In 1985, when we adopted the new staging system for lung cancer, it was not difficult for us to quickly adapt it to all reports. In fact, the two American thoracic surgical journals agreed that within 1 year the new staging system had to be used in all reported results of lung cancer. I would urge that everybody reporting their results of mesothelioma use this new staging system, which does seem to apply and is similar to the TNM staging system that was proposed with some modifications. It would be worthwhile for our journals to accept only articles reported in the new staging system. All of the data that Dr. Rusch analyzed were restaged from the pathology reports. It is not that difficult to analyze the surgical staging and the pathologic staging from these reports and reclassify them according to this new staging system.

Dr. Rusch. Thank you for the endorsement. I would emphasize that the IMIG does not consider this a perfect staging system. We realize this is a first pass. We hope that the application of this staging system both retrospectively and prospectively will enable us to refine it just as has been done for non-small-cell cancer. 